\title{
A NOTE ON QUOTIENT SEMIRINGS
}

\author{
D. R. LATORRE
}

In a previous article [1] Allen obtained an exact analogue of the fundamental homomorphism theorem for a certain class of semiring homomorphisms called maximal homomorphisms. The crux of the argument involved the notion of a $Q$-ideal, by which the semiring is partitioned into cosets modulo such an ideal, and the construction of a particular quotient semiring modulo a $Q$-ideal. This note gives necessary and sufficient conditions, in terms of homomorphisms, for an ideal to be a $Q$-ideal; shows that $Q$-ideals are included among the $k$-ideals considered in [4]-[7]; and deduces that the particular quotient semiring modulo a $Q$-ideal used in [1] actually coincides with the more familiar quotient structure first employed in [2]-[3]. We use without comment the definitions, terminology, and notation of $[1]$.

The familiar construction of a quotient semiring modulo an ideal is as follows: given an ideal $I$ of semiring $(S,+, \cdot)$ define a relation $\rho$ by

$$
\rho=\left\{(x, y) \in S \times S: x+i_{1}=y+i_{2} \text { for some } i_{1}, i_{2} \in I\right\} .
$$

Then $\rho$ is a congruence on both $(S,+)$ and $(S, \cdot)$ and under the usual operations of addition $(\oplus)$ and multiplication (o) of congruence classes the $\rho$-classes become a semiring $(S / I, \oplus, 0)$. The $\rho$-class containing $a \in S$ need not be the coset $a+I$. From [5] we note that $I$ is contained in a $\rho$-class $C_{I}$ which is the smallest $k$-ideal of $S$ containing $I$ and the zero of $(S / I, \oplus, 0)$, that $(S / I, \oplus, 0)=\left(S / C_{I}, \oplus, 0\right)$, and that $I$ is a $\rho$-class if and only if $I$ is a $k$-ideal. (An ideal is a $k$-ideal if whenever $x+i \in I$, where $x \in S$ and $i \in I$, we have $x \in I$.)

Theorem 1. Let $I$ be a $Q$-ideal of semiring $(S,+, \cdot)$ and define a relation $\eta$ by

$$
\eta=\{(x, y) \in S \times S: x, y \in q+I \text { for some } q \in Q\} .
$$

Then $\eta=\rho$ and $I$ is the $\rho$-class containing zero.

Proof. If $(x, y) \in \eta$, say $x=q+i_{1}, y=q+i_{2}$ for some $q \in Q$ and $i_{1}$, $i_{2} \in I$, then $x+i_{2}=\left(q+i_{1}\right)+i_{2}=\left(q+i_{2}\right)+i_{1}=y+i_{1}$ so that $(x, y) \in \rho$. Conversely, suppose $(x, y) \in \rho$, say $x+i_{1}=y+i_{2}$ with $i_{1}, i_{2} \in I$. Let $x=q_{1}+i_{3}$ and $y=q_{2}+i_{4}$ where $q_{1}, q_{2} \in Q$ and $i_{3}, i_{4} \in I$. Then

Received by the editors May 30, 1969. 


$$
\begin{aligned}
x+i_{1} & =\left(q_{1}+i_{3}\right)+i_{1}=q_{1}+\left(i_{3}+i_{1}\right)=y+i_{2}=\left(q_{2}+i_{4}\right)+i_{2} \\
& =q_{2}+\left(i_{4}+i_{2}\right) .
\end{aligned}
$$

But $q_{1}+\left(i_{3}+i_{1}\right) \in q_{1}+I$ and $q_{2}+\left(i_{4}+i_{2}\right) \in q_{2}+I$, whence $q_{1}=q_{2}$ and $q_{1}+I=q_{2}+I$. Thus $(x, y) \in \eta$.

Now there is a unique $q \in Q$ with $0 \in q+I$, say $0=q+i$, so $q+I$ $=q+0+I=q+q+i+I \subseteq q+q+I$. By Lemma 7 of [1] $q+q+I$ is contained in a unique coset $q^{\prime}+I\left(q^{\prime} \in Q\right)$, which, in view of $q+I$ $\subseteq q+q+I$, is $q+I$. Thus $q+q+I=q+I$ implies $q+q=q+i_{1}$, for some $i_{1} \in I$. Then $I=0+I=q+i+I \subseteq q+I$ and

$$
\begin{aligned}
q+I & =q+0+I=q+q+i+I=q+i_{1}+i+I \\
& =0+i_{1}+I \subseteq I
\end{aligned}
$$

which shows that $I$ is the $\eta$-class $q+I$ containing 0 .

Theorem 1 and the preceding remarks immediately give

Corollary 2. A Q-ideal I of semiring $(S,+, \cdot)$ is a $k$-ideal and the zero of the quotient semiring $(S / I, \oplus, 0)$.

REMARK. From Theorem 1 it follows that there is at most one partition of $S$ by cosets of $I$; an independent proof of this without introducing relation $\rho$ is straightforward.

TheOREM 3. Let I be a $Q$-ideal of a semiring $(S,+, \cdot)$, let $(S / I, \oplus, 0)$ be the quotient semiring defined above, and let $\left(\{q+I\}_{q \in Q}, \oplus_{Q}, \odot_{Q}\right)$ be the quotient semiring of [1]. Then these two quotient semirings are equal.

Proof. The set of $\eta$-classes is precisely the set $\{q+I\}_{q \in Q}$ and $\oplus_{Q}$ and $\odot_{Q}$ are ordinary addition and multiplication of $\eta$-classes. Theorem 1 shows $\eta=\rho$.

REMARK. Theorem 3 shows that the isomorphism of Theorem 9 in [1] can be replaced by equality.

Theorem 4. An ideal I of a semiring $(S,+, \cdot)$ is a $Q$-ideal for some subset $Q$ of $S$ if and only if there is a semiring homomorphism $\phi: S \rightarrow T$ such that the inverse image of each $t \in T$ is a coset of $I$.

Proof. If $I$ is a $Q$-ideal for the subset $Q$ of $S$, the natural homomorphism $\phi: S \rightarrow S / I$ is such that $\phi^{-1}(q+I)=q+I$. Conversely, suppose $\phi: S \rightarrow T$ is such a semiring homomorphism. Define $\eta$ on $S$ by $\eta=\{(x, y) \in S \times S: x \phi=y \phi\}$. Then $\eta$ is a congruence on $(S,+, \cdot)$ and each $\eta$-class is a coset of $I$. The partition of $S$ consisting of the distinct $\eta$-classes makes $I$ into a $Q$-ideal.

In [1] a homomorphism $\phi: S \rightarrow S^{\prime}$ is called maximal if for each 
$a \in S^{\prime}$ there is an element $c_{a} \in \eta^{-1}(\{a\})$ such that $x+\operatorname{ker} \eta \subseteq c_{a}+\operatorname{ker} \eta$ for each $x \in \eta^{-1}(\{a\})$. Thus for each $a \in S^{\prime}, \eta^{-1}(\{a\})=c_{a}+$ ker $\eta$. Conversely, if $\eta: S \rightarrow S^{\prime}$ is any homomorphism such that for each $a \in S^{\prime}, \eta^{-1}(\{a\})=c_{a}+$ ker $\eta$ for some $c_{a} \in S$ then $\eta$ is maximal. In short, a homomorphism $\eta: S \rightarrow S^{\prime}$ is maximal if and only if the inverse image of each $a \in S^{\prime}$ is a coset of ker $\eta$. Together with Theorem 4 this immediately gives (see Lemma 14 of [1])

COROLlary 5. The kernel of a maximal homomorphism is a Q-ideal.

\section{REFERENCES}

1. Paul J. Allen, A fundamental theorem of homomorphisms for semirings, Proc. Amer. Math. Soc. 21 (1969), 412-416.

2. S. Bourne, The Jacobson radical of a semiring, Proc. Nat. Acad. Sci. U.S.A. 37 (1951), 163-170. MR 13, 7.

3. - On the homomorphism theorem for semirings, Proc. Nat. Acad. Sci. U.S.A. 38 (1952), 118-119. MR 13, 618.

4. M. Henriksen, Ideals in semirings with commutative addition, Notices Amer. Math. Soc. 5 (1958), 321. Abstract \#542-183.

5. D. R. LaTorre, On $h$-ideals and $k$-ideals in hemirings, Publ. Math. Debrecen 12 (1965), 219-226. MR 34 \#214.

6. - A note on the Jacobson radical of a hemiring, Publ. Math. Debrecen 14 (1967), 9-13. MR 36 \#3827.

7. - The Brown-McCoy radicals of a hemiring, Publ. Math. Debrecen 14 (1967), 15-28. MR 36 \#3828.

\section{Clemson University}

